\title{
Research Article On the Barnes' Type Related to Multiple Genocchi Polynomials on $\mathbb{Z}_{p}$
}

\author{
J. Y. Kang, H. Y. Lee, N. S. Jung, and C. S. Ryoo \\ Department of Mathematics, Hannam University, Daejeon 306-791, Republic of Korea \\ Correspondence should be addressed to J. Y. Kang, rkdwjddnr2002@yahoo.co.kr
}

Received 29 July 2012; Revised 17 August 2012; Accepted 21 August 2012

Academic Editor: Józef Banaś

Copyright (C) 2012 J. Y. Kang et al. This is an open access article distributed under the Creative Commons Attribution License, which permits unrestricted use, distribution, and reproduction in any medium, provided the original work is properly cited.

\begin{abstract}
Using fermionic $p$-adic invariant integral on $\mathbb{Z}_{p}$, we construct the Barnes' type multiple Genocchi numbers and polynomials. From those numbers and polynomials, we derive the twisted Barnes' type multiple Genocchi numbers and polynomials. Moreover, we will find the Barnes' type multiple Genocchi zeta function.
\end{abstract}

\section{Introduction}

Recently, theoretical physicists have devised ultrametric structures similar to tree-like structures arising in the study of physical systems because the fact is that the physical space may no longer be Archimedean seems plausible to some mathematical physicists at a very small distance. They have looked for construct-related models using $p$-adic numbers and $p$-adic analysis. So, $p$-adic numbers are used in mathematical physics (in particular string theory, field theory) as well as in other areas of natural sciences which complicated fractal behavior and hierarchical structures. Also, $q$-Volkenborn integral which is made by Kim is used in the functional equation of the $q$-zeta function, the $q$-Stirling numbers, and Mahler theory of integration with respect to the ring $\mathbb{Z}_{p}$ together with Iwasawas $p$-adic $q-L$ function. So we study the $q$-Gnocchi numbers and polynomials in $q$-type of special generating functions (see $[1,2]$ ).

Let $p$ be a fixed odd prime number. Throughout this paper, $\mathbb{Z}_{p}, \mathbb{Q}_{p}, \mathbb{C}$, and $\mathbb{C}_{p}$ denote the ring of $p$-adic rational integers, the field of $p$-adic rational numbers, the complex number field, and the completion of the algebraic closure of $\mathbb{Q}_{p}$, respectively. Let $\mathbb{N}$ be the set of natural numbers and $\mathbb{Z}^{+}=\mathbb{N} \cup\{0\}$. Let $v_{p}$ be the normalized exponential valuation of $\mathbb{C}_{p}$ with $|p|_{p}=$ $p^{-v_{p}(p)}=1 / p(\operatorname{see}[1-15])$. 
Let $d$ be a fixed integer and let $p$ be a fixed prime number. For any positive integer $N$, we set

$$
\begin{gathered}
X=X_{d}=\lim _{\overleftarrow{N}}\left(\frac{\mathbb{Z}}{d p^{N} \mathbb{Z}}\right), \quad X_{1}=\mathbb{Z}_{p}, \\
X^{*}=\bigcup_{\substack{0<a<d p \\
(a, p)=1}}\left(a+d p \mathbb{Z}_{p}\right), \\
a+d p^{N} \mathbb{Z}_{p}=\left\{x \in X \mid x \equiv a\left(\bmod d p^{N}\right)\right\},
\end{gathered}
$$

where $a \in \mathbb{Z}$ lies in $0 \leq a<d p^{N}$.

We say that $f: \mathbb{Z}_{p} \rightarrow \mathbb{C}_{p}$ is uniformly differentiable function at a point $a \in \mathbb{Z}_{p}$, and we write $f \in \mathrm{UD}\left(\mathbb{Z}_{p}\right)$ if the difference quotients $\Phi_{f}: \mathbb{Z}_{p} \times \mathbb{Z}_{p} \rightarrow \mathbb{C}_{p}$ such that

$$
\Phi_{f}(x, y)=\frac{f(x)-f(y)}{x-y}
$$

have a limit $f^{\prime}(a)$ as $(x, y) \rightarrow(a, a)$.

As well-known definition, the Genocchi polynomials are defined by

$$
\begin{gathered}
F(t)=\frac{2 t}{e^{t}+1}=e^{G t}=\sum_{n=0}^{\infty} G_{n} \frac{t^{n}}{n !}, \\
F(t, x)=\frac{2 t}{e^{t}+1} e^{x t}=e^{G(x) t}=\sum_{n=0}^{\infty} G_{n}(x) \frac{t^{n}}{n !},
\end{gathered}
$$

with the usual convention of replacing $G^{n}(x)$ by $G_{n}(x)$. In the special case, $x=0, G_{n}(0)=G_{n}$ are called the $n$th Genocchi numbers.

For $f \in \mathrm{UD}\left(\mathbb{Z}_{p}\right)$, Kim defined the $q$-deformed fermionic $p$-adic integral on $Z_{p}$,

$$
I_{-q}(f)=\int_{\mathbb{Z}_{p}} f(x) d \mu_{-q}(x)=\lim _{N \rightarrow \infty} \frac{1}{\left[p^{N}\right]_{-q}} \sum_{x=0}^{p^{N}-1} f(x)(-q)^{x} .
$$

Note that

$$
I_{-1}(f)=\lim _{q \rightarrow 1} I_{-q}(f)
$$

If we take $f(x)=t e^{t x}$, by (1.4), we see that

$$
\int_{\mathbb{Z}_{p}} t e^{t x} d \mu_{-1}(x)=\frac{2 t}{1+e^{t}}=\sum_{n=0}^{\infty} G_{n} \frac{t^{n}}{n !}
$$


By the same method, we note that

$$
\int_{\mathbb{Z}_{p}} t e^{t(x+y)} d \mu_{-1}(x)=\frac{2 t}{1+e^{t}} e^{t x}=\sum_{n=0}^{\infty} G_{n}(x) \frac{t^{n}}{n !}
$$

And note that

$$
\int_{\mathbb{Z}_{p}} f(x) d \mu_{-1}(x)=\int_{X} f(x) d \mu_{-1}(x) .
$$

Barnes' type multiple zeta function $\zeta_{N}\left(s, w \mid a_{1}, \ldots, a_{N}\right)$ depends on the parameters $a_{1}, \ldots, a_{N}$ that will be assumed to be positive. It is defined by

$$
\zeta_{N}\left(s, w \mid a_{1}, \ldots, a_{N}\right)=\sum_{m_{1}, \ldots, m_{N}=0}^{\infty}\left(w+m_{1} a_{1}+\cdots+m_{N} a_{N}\right)^{-s}
$$

for $\operatorname{Re}(s)>N, \operatorname{Re}(w)>0$. Barnes showed that $\zeta_{N}$ had a meromorphic continuation in $s$ (with simple pole only at $s=1, \ldots, N)$ and defined his multiple gamma function $\Gamma_{N}(w)$ in terms of the $s$-derivative at $s=0$, which may be recalled here as follows:

$$
\Psi_{n}\left(w \mid a_{1}, \ldots, a_{N}\right)=\partial_{s} \zeta_{N}\left(s, w \mid a_{1} \ldots, a_{N}\right) \mid s=0
$$

\section{Barnes' Type Multiple Genocchi Polynomials}

In this section, we assume that $w_{1}, \ldots, w_{r} \in \mathbb{Z}_{p}$. For $x \in \mathbb{Q}^{+}, r \in \mathbb{N}$, we define Barnes' type multiple Genocchi polynomials as follows:

$$
\begin{aligned}
& \underbrace{\int_{\mathbb{Z}_{p}} \cdots \int_{\mathbb{Z}_{p}} t^{r} e^{\left(x+w_{1} y_{1}+\cdots+w_{r} y_{r}\right) t} d \mu_{-1}\left(y_{1}\right) \cdots d \mu_{-1}\left(y_{r}\right)}_{r \text {-times }} \\
& =\frac{2^{r} t^{r}}{\left(1+e^{t w_{1}}\right)\left(1+e^{t w_{2}}\right) \cdots\left(1+e^{t w w_{r}}\right)} e^{t x} \\
& =\sum_{n=0}^{\infty} G_{n}^{(r)}\left(x \mid w_{1}, \ldots, w_{r}\right) \frac{t^{n}}{n !} .
\end{aligned}
$$

In the special case, $x=0, G_{n}^{(r)}\left(w_{1}, \ldots, w_{r}\right)=G_{n}^{(r)}\left(0 \mid w_{1}, \ldots, w_{r}\right)$ are called the $n$th Barnes' type multiple Genocchi numbers. 
Thus, we have

$$
\begin{gathered}
G_{0}^{(r)}\left(x \mid w_{1}, \ldots, w_{r}\right)=G_{1}^{(r)}\left(x \mid w_{1}, \ldots, w_{r}\right)=\cdots=G_{r-1}^{(r)}\left(x \mid w_{1}, \ldots, w_{r}\right)=0, \\
\frac{G_{n+r}^{(r)}\left(x \mid w_{1}, \ldots, w_{r}\right)}{\left(\begin{array}{c}
n+r \\
r
\end{array}\right) r !}=\underbrace{\int_{\mathbb{Z}_{p}} \cdots \int_{\mathbb{Z}_{p}}\left(x+w_{1} y_{1}+\cdots+w_{r} y_{r}\right)^{n} d \mu_{-1}\left(y_{1}\right) \cdots d \mu_{-1}\left(y_{r}\right) .}_{r \text {-times }}
\end{gathered}
$$

For $r \geq 1, n>0$, we can readily prove the following (2.3) from (2.2):

$$
G_{n+r}^{(r)}\left(x \mid w_{1}, \ldots, w_{r}\right)=\sum_{l=0}^{n}\left(\begin{array}{c}
n+r \\
l+r
\end{array}\right) x^{n-l} G_{l+r}^{(r)}\left(w_{1}, \ldots, w_{r}\right)
$$

where $\left(\begin{array}{c}n+r \\ l+r\end{array}\right)$ is a binomial coefficient.

Theorem 2.1 (Property of distribution of $G_{n}^{(r)}\left(x \mid w_{1}, \ldots, w_{r}\right)$ ). For $r \geq 1, n>0$ and $m \in \mathbb{N}$ with $m \equiv 1(\bmod 2)$,

$$
G_{n}^{(r)}\left(x \mid w_{1}, \ldots, w_{r}\right)=\frac{m^{n}}{m^{r}} \sum_{n_{1}, \ldots, n_{r}=0}^{m-1}(-1)^{\sum_{i=1}^{r} n_{i}} G_{n}^{(r)}\left(\frac{x+\sum_{i=1}^{r} w_{i} n_{i}}{m} \mid w_{1}, \ldots, w_{r}\right) .
$$

Let $x$ be Dirichlet's character with conductor $d \in \mathbb{N}$ with $d \equiv 1(\bmod 2)$ :

$$
\begin{aligned}
F^{(r)}\left(t, x \mid w_{1}, \ldots, w_{r}\right) & =\frac{2^{r} t^{r}}{\prod_{i=1}^{r}\left(1+e^{t w_{i}}\right)} e^{t x} \\
& =\sum_{n=0}^{\infty} G_{n}^{(r)}\left(x \mid w_{1}, \ldots, w_{r}\right) \frac{t^{n}}{n !} .
\end{aligned}
$$

By (2.1) and (2.5), we see that

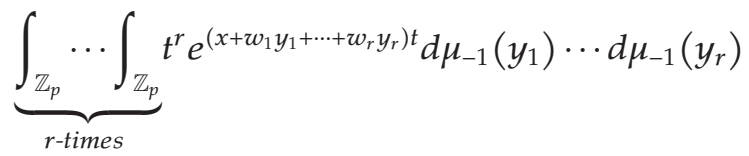

$$
\begin{aligned}
& =2^{r} t^{r} \sum_{m_{1}, \ldots, m_{r}=0}^{\infty}(-1)^{m_{1}+\cdots+m_{r}} e^{\left(x+w_{1} m_{1}+\cdots+w_{r} m_{r}\right) t} .
\end{aligned}
$$


From (2.2) and (2.6), we have

$$
\begin{aligned}
\frac{G_{n+r}^{(r)}\left(x \mid w_{1}, \ldots, w_{r}\right)}{\left(\begin{array}{c}
n+r \\
r
\end{array}\right) r !} & =\underbrace{\int_{\mathbb{Z}_{p}} \cdots \int_{\mathbb{Z}_{p}}}_{r \text {-times }}\left(x+w_{1} y_{1}+\cdots+w_{r} y_{r}\right)^{n} d \mu_{-1}\left(y_{1}\right) \cdots d \mu_{-1}\left(y_{r}\right) \\
& =2^{r} \sum_{m_{1}, \ldots, m_{r}=0}^{\infty}(-1)^{m_{1}+\cdots+m_{r}}\left(x+w_{1} m_{1}+\cdots+w_{r} m_{r}\right)^{n}
\end{aligned}
$$

By (2.5), we see that

$$
\begin{aligned}
F_{X}^{(r)}\left(t, x \mid w_{1}, \ldots, w_{r}\right) & =\frac{2^{r} t^{r}\left(\sum_{a=0}^{d-1}(-1)^{a} X(a) e^{w_{1} a t}\right) \cdots\left(\sum_{a=0}^{d-1}(-1)^{a} X(a) e^{w_{r} a t}\right)}{\left(1+e^{w_{1} d t}\right) \cdots\left(1+e^{w_{r} d t}\right)} e^{t x} \\
& =2^{r} t^{r} \sum_{m_{1}, \ldots, m_{r}=0}^{\infty}(-1)^{m_{1}+\cdots+m_{r}}\left(\prod_{i=1}^{r} \chi\left(m_{i}\right)\right) e^{\left(x+w_{1} m_{1}+\cdots+w_{r} m_{r}\right) t}
\end{aligned}
$$

We define the generalized Barnes' type multiple Genocchi polynomials attached to $x$ as follows: for $m \geq 0$ and $w_{i} \in \mathbb{Z}_{p}(i=1,2, \ldots, n)$,

$$
\begin{gathered}
G_{0, x}^{(r)}\left(x \mid w_{1}, \ldots, w_{r}\right)=G_{1, X}^{(r)}\left(x \mid w_{1}, \ldots, w_{r}\right)=\cdots=G_{r-1, x}^{(r)}\left(x \mid w_{1}, \ldots, w_{r}\right)=0, \\
\frac{G_{n+r}^{(r)}\left(x \mid w_{1}, \ldots, w_{r}\right)}{\left(\begin{array}{c}
n+r \\
r
\end{array}\right) r !}=\underbrace{\int_{X} \ldots \int_{X}}_{r \text {-times }}\left(\prod_{i=1}^{r} x\left(x_{i}\right)\right)\left(x+\sum_{i=1}^{r} w_{i} y_{i}\right)^{n} d \mu_{-1}\left(y_{1}\right) \cdots d \mu_{-1}\left(y_{r}\right) .
\end{gathered}
$$
theorem.

For simple calculation of fermionic $p$-adic invariant on $\mathbb{Z}_{p}$, we note the following Theorem 2.2 (Property of distribution of $G_{n, x}^{(r)}\left(x \mid w_{1}, \ldots, w_{r}\right)$ ).

$$
G_{n, X}^{(r)}\left(x \mid w_{1}, \ldots, w_{r}\right)=\frac{d^{n}}{d^{r}} \sum_{n_{1}, \ldots, n_{r}=0}^{d-1}(-1)^{\sum_{i=1}^{r} n_{i}} G_{n, X}^{(r)}\left(\frac{x+\sum_{i=1}^{r} w_{i} n_{i}}{d} \mid w_{1}, \ldots, w_{r}\right)
$$

Theorem 2.2 is distribution relation for the generalized Barnaes' type multiple Genocchi polynomials attached to $x$. In the special case, $x=0, G_{n, X}^{(r)}\left(w_{1}, \ldots, w_{r}\right)=G_{n, X}^{(r)}(0 \mid$ $\left.w_{1}, \ldots, w_{r}\right)$ are called the generalized Barnes' type multiple Genocchi numbers attached to $X$.

By (2.8) and (2.9), we get

$$
\frac{G_{n+r, X}^{(r)}\left(x \mid w_{1}, \ldots, w_{r}\right)}{\left(\begin{array}{c}
n+r \\
r
\end{array}\right) r !}=2^{r} \sum_{m_{1}, \ldots, m_{r}=0}^{\infty}(-1)^{\sum_{i=1}^{r} m_{i}}\left(\prod_{i=1}^{r} \chi\left(m_{i}\right)\right)\left(x+\sum_{i=1}^{r} w_{i} m_{i}\right)^{n} .
$$




\section{Twisted Barnes' Type Multiple Genocchi Polynomials}

Let $T_{p}=\lim _{N \rightarrow \infty} C_{p^{N}}=\cup_{N \geq 0} C_{p^{N}}$, where $C_{p^{N}}=\left\{\zeta \mid \zeta^{p^{N}}=1\right\}$ is the cyclic group of order $p^{N}$. For $\varepsilon \in T_{p}$, we denote by $\phi_{\varepsilon}: \mathbb{Z}_{p} \rightarrow \mathbb{C}_{p}$ the locally constant function $x \mapsto \phi_{\varepsilon}(x)=\varepsilon^{x}$. If we take $f(x)=\phi_{\varepsilon}(x) e^{t x}$, then we get

$$
\int_{\mathbb{Z}_{p}} t \phi_{\varepsilon}(x) e^{t x} d \mu_{-1}(x)=\frac{2 t}{1+\varepsilon e^{t}}
$$

By (3.1), we easily see that

$$
\int_{X} t \phi_{\varepsilon}(x) X(x) e^{t x} d \mu_{-1}(x)=\frac{2 t \sum_{a=0}^{d-1}(-1)^{a} \varepsilon^{a} X(a) e^{a t}}{1+\varepsilon^{d} e^{d t}}
$$

From (3.1) and (3.2), we define the twisted Genocchi numbers and the generalized twisted Genocchi numbers attached to $X$ as follows:

$$
\frac{2 t}{1+\varepsilon e^{t}}=\sum_{n=0}^{\infty} G_{n, e} \frac{t^{n}}{n !}, \quad \frac{2 t \sum_{a=0}^{d-1}(-1)^{a} \varepsilon^{a} X(a) e^{a t}}{1+\varepsilon^{d} e^{d t}}=\sum_{n=0}^{\infty} G_{n, X, \varepsilon} \frac{t^{n}}{n !} .
$$

In (3.1), (3.2), and (3.3), we get

$$
\int_{\mathbb{Z}_{p}} \phi_{\varepsilon}(x) x^{n} d \mu_{-1}(x)=\frac{G_{n+1, \varepsilon}}{n+1}, \quad \int_{X} \phi_{\varepsilon}(x) X(x) x^{n} d \mu_{-1}(x)=\frac{G_{n+1, x, \varepsilon}}{n+1} .
$$

By using fermionic multivariate $p$-adic invariant integral on $\mathbb{Z}_{p}$, we define the twisted Barnes' type multiple Genocchi polynomials as follows:

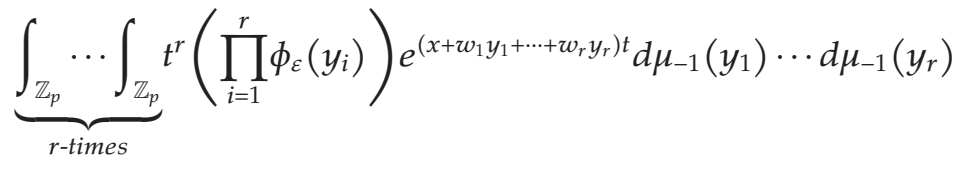

$$
\begin{aligned}
& =\frac{2^{r} t^{r}}{\left(1+\varepsilon e^{w_{1} t}\right) \cdots\left(1+\varepsilon e^{w_{r} t}\right)} e^{t x} \\
& =\sum_{n=0}^{\infty} G_{n, \varepsilon}^{(r)}\left(x \mid w_{1}, \ldots, w_{r}\right) \frac{t^{n}}{n !}, \quad \varepsilon \in T_{p} .
\end{aligned}
$$

$G_{n, \varepsilon}^{(r)}\left(x \mid w_{1}, \ldots, w_{r}\right)$ are called the twisted Barnes' type multiple Genocchi polynomials. In the special case, $x=0, G_{n, \varepsilon}^{(r)}\left(w_{1}, \ldots, w_{r}\right)=G_{n, \varepsilon}^{(r)}\left(0 \mid w_{1}, \ldots, w_{r}\right)$ are called the twisted Barnes' type multiple Genocchi numbers. 
From (3.5), we can derive the following:

$$
\begin{aligned}
& \underbrace{\int_{\mathbb{Z}_{p}} \cdots \int_{\mathbb{Z}_{p}} t^{r}}_{r \text {-times }}\left(\prod_{i=1}^{r} \phi_{\varepsilon}\left(y_{i}\right)\right)\left(x+w_{1} y_{1}+\cdots+w_{r} y_{r}\right)^{n} d \mu_{-1}\left(y_{1}\right) \cdots d \mu_{-1}\left(y_{r}\right) \\
& \quad=\frac{G_{n+r, \varepsilon}^{(r)}\left(x \mid w_{1}, \ldots, w_{r}\right)}{\left(\begin{array}{c}
n+r \\
r
\end{array}\right) r !} .
\end{aligned}
$$

Let $\chi$ be the primitive Drichlet character with conductor $d \in \mathbb{N}$ with $d \equiv 1(\bmod 2)$. Then we define the generalized twisted Barnes' type multiple Genocchi numbers attached to $x$ :

$$
\begin{gathered}
\underbrace{\int_{\mathbb{Z}_{p}} \cdots \int_{\mathbb{Z}_{p}} t^{r}\left(\prod_{i=1}^{r} \phi_{\varepsilon}\left(x_{i}\right)\right)\left(\prod_{i=1}^{r} \chi\left(x_{i}\right)\right) e^{\left(w_{1} x_{1}+\cdots+w_{r} x_{r}\right) t} d \mu_{-1}\left(x_{1}\right) \cdots d \mu_{-1}\left(x_{r}\right)}_{r \text {-times }} \\
=\frac{2^{r} t^{r}\left(\sum_{l=0}^{d-1}(-1)^{l} \varepsilon^{l} \chi(l) e^{w_{1} l t}\right) \cdots\left(\sum_{l=0}^{d-1}(-1)^{l} \varepsilon^{l} \chi(l) e^{w_{r} l t}\right)}{\left(1+\varepsilon^{d} e^{w_{1} d t}\right) \cdots\left(1+\varepsilon^{d} e^{w_{r} d t}\right)} \\
=\sum_{n=0}^{\infty} G_{n, \varepsilon, X}^{(r)}\left(w_{1}, \ldots, w_{r}\right) \frac{t^{n}}{n !}=F_{\varepsilon, X}^{(r)}\left(t \mid w_{1}, \ldots, w_{r}\right) .
\end{gathered}
$$

From (3.7), we see that

$$
F_{\varepsilon, X}^{(r)}\left(t \mid w_{1}, \ldots, w_{r}\right)=2^{r} t^{r} \sum_{m_{1}, \ldots, m_{r}=0}^{\infty}(-1)^{\sum_{i=1}^{r} m_{i} \varepsilon \sum_{i=1}^{r} m_{i}}\left(\prod_{i=1}^{r} \chi\left(m_{i}\right)\right) e^{t\left(\sum_{i=1}^{r} w_{i} m_{i}\right)}
$$

By using (3.7), we easily see that

$$
\begin{aligned}
& \frac{G_{n, \varepsilon, X}^{(r)}\left(w_{1}, \ldots, w_{r}\right)}{\left(\begin{array}{c}
n+r \\
r
\end{array}\right) r !} \\
& \quad=\underbrace{\int_{X} \cdots \int_{X}}_{r \text {-times }}\left(\prod_{i=1}^{r} \phi_{\varepsilon}\left(x_{i}\right)\right)\left(\prod_{i=1}^{r} X\left(x_{i}\right)\right)\left(w_{1} x_{1}+\cdots+w_{r} x_{r}\right)^{n} d \mu_{-1}\left(x_{1}\right) \cdots d \mu_{-1}\left(x_{r}\right) .
\end{aligned}
$$


Theorem 3.1 (Property of distribution of $G_{n, \varepsilon, x}^{(r)}\left(x \mid w_{1}, \ldots, w_{r}\right)$ ).

$$
\begin{aligned}
G_{n, \varepsilon, X}^{(r)}\left(x \mid w_{1}, \ldots, w_{r}\right) \\
\quad=\frac{d^{n}}{d^{r}} \sum_{n_{1}, \ldots, n_{r}=0}^{d-1}(-1)^{\sum_{i=1}^{r} n_{i} \varepsilon \sum_{i=1}^{r} n_{i}}\left(\prod_{i=1}^{r} \chi\left(n_{i}\right)\right) G_{n, \varepsilon^{d}}^{(r)}\left(\frac{x+\sum_{i=1}^{r} w_{i} n_{i}}{d} \mid w_{1}, \ldots, w_{r}\right) .
\end{aligned}
$$

\section{Remark}

Let $w_{1}, \ldots, w_{r}$ be taken nonnegative in complex plane. We consider the Barnes' type multiple Genocchi zeta function $\zeta\left(s, x \mid w_{1}, \ldots, w_{r}\right)$ as follows:

$$
\zeta\left(s, x \mid w_{1}, \ldots, w_{r}\right)=2^{r} \sum_{m_{1}, \ldots, m_{r}=0}^{\infty} \frac{(-1)^{m_{1}+\cdots+m_{r}}}{\left(x+w_{1} m_{1}+\cdots+w_{r} m_{r}\right)^{s}}
$$

where $s \in \mathbb{C}$, and $\operatorname{Re}(x)>0$.

By (4.1) and (2.7), we get

$$
\zeta\left(-n, x \mid w_{1}, \ldots, w_{r}\right)=\frac{G_{n+r}^{(r)}\left(x \mid w_{1}, \ldots, w_{r}\right)}{\left(\begin{array}{c}
n+r \\
r
\end{array}\right) r !}
$$

where $n \in \mathbb{Z}^{+}$.

Define

$$
L_{X}\left(s, x \mid w_{1}, \ldots, w_{r}\right)=2^{r} \sum_{m_{1}, \ldots, m_{r}=0}^{\infty} \frac{(-1)^{m_{1}+\cdots+m_{r}}\left(\prod_{i=1}^{r} X\left(m_{i}\right)\right)}{\left(x+w_{1} m_{1}+\cdots+w_{r} m_{r}\right)^{s}}
$$

where $s \in \mathbb{C}$, and $\operatorname{Re}(x)>0$.

From (4.3), we note that

$$
L_{X}\left(-n, x \mid w_{1}, \ldots, w_{r}\right)=\frac{G_{n+r, X}^{(r)}\left(x \mid w_{1}, \ldots, w_{r}\right)}{\left(\begin{array}{c}
n+r \\
r
\end{array}\right) r !}, \quad n \in \mathbb{Z}^{+}
$$

\section{References}

[1] T. Kim, " $q$-Volkenborn integration," Russian Journal of Mathematical Physics, vol. 9, no. 3, pp. 288-299, 2002.

[2] T. Kim, "On Euler-Barnes multiple zeta functions," Russian Journal of Mathematical Physics, vol. 10, no. 3, pp. 261-267, 2003.

[3] T. Kim, "Barnes-type multiple q-zeta functions and q-Euler polynomials," Journal of Physics A, vol. 43, no. 25,11 pages, 2010.

[4] T. Kim, "An invariant $p$-adic $q$-integral on $\mathbb{Z}_{p}$, " Applied Mathematics Letters, vol. 21, no. 2, pp. 105-108, 2008.

[5] L.-C. Jang, T. Kim, D.-H. Lee, and D.-W. Park, "An application of polylogarithms in the analogs of Genocchi numbers," Notes on Number Theory and Discrete Mathematics, vol. 7, no. 3, pp. 65-69, 2001. 
[6] L.-C. Jang, "On a $q$-analogue of the $p$-adic generalized twisted $L$-functions and $p$-adic $q$-integrals," Journal of the Korean Mathematical Society, vol. 44, no. 1, pp. 1-10, 2007.

[7] L.-C. Jang, "A study on the distribution of twisted q-Genocchi polynomials," Advanced Studies in Contemporary Mathematics, vol. 18, no. 2, pp. 181-189, 2009.

[8] C. S. Ryoo, "On the generalized Barnes type multiple $q$-Euler polynomials twisted by ramified roots of unity," Proceedings of the Jangjeon Mathematical Society, vol. 13, no. 2, pp. 255-263, 2010.

[9] C. S. Ryoo, "On the generalized Barnes type multiple q-Euler polynomials," Proceedings of the Jangjeon Mathematical Society, vol. 13, no. 3, pp. 327-335, 2010.

[10] V. Kurt and M. Cenkci, "A new approach to $q$-Genocchi numbers and polynomials," Bulletin of the Korean Mathematical Society, vol. 47, no. 3, pp. 575-583, 2010.

[11] Y. Simsek, "On $p$-adic twisted $q$-L-functions related to generalized twisted Bernoulli numbers," Russian Journal of Mathematical Physics, vol. 13, no. 3, pp. 340-348, 2006.

[12] S.-H. Rim and T. Kim, "A note on $p$-adic Euler measure on $\mathbb{Z}_{p}$," Russian Journal of Mathematical Physics, vol. 13, no. 3, pp. 358-361, 2006.

[13] S.-H. Rim, K. H. Park, and E. J. Moon, “On Genocchi numbers and polynomials," Abstract and Applied Analysis, vol. 2008, Article ID 898471, 7 pages, 2008.

[14] T. Kim, "A note on $q$-Volkenborn integration," Proceedings of the Jangjeon Mathematical Society, vol. 8 , no. 1, pp. 13-17, 2005.

[15] M. Domaratzki, “Combinatorial interpretations of a generalization of the Genocchi numbers," Journal of Integer Sequences, vol. 7, 2004. 


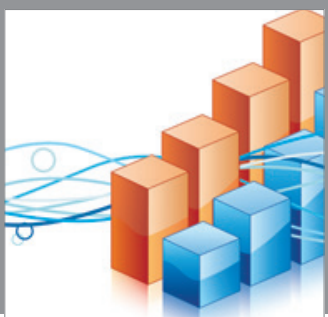

Advances in

Operations Research

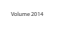

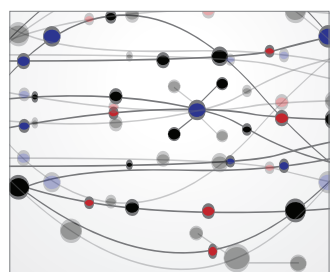

\section{The Scientific} World Journal
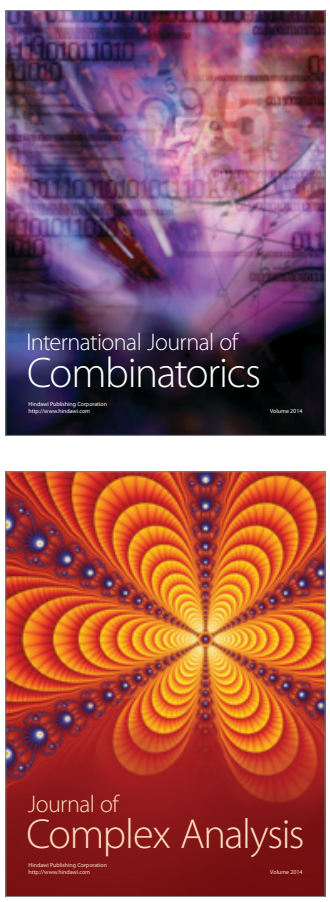

International Journal of

Mathematics and

Mathematical

Sciences
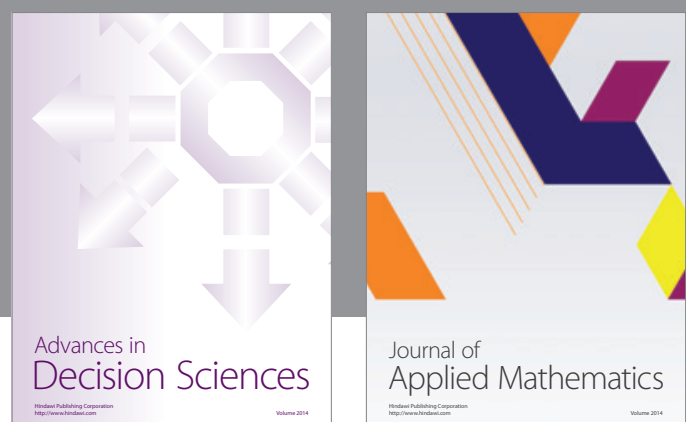

Journal of

Applied Mathematics
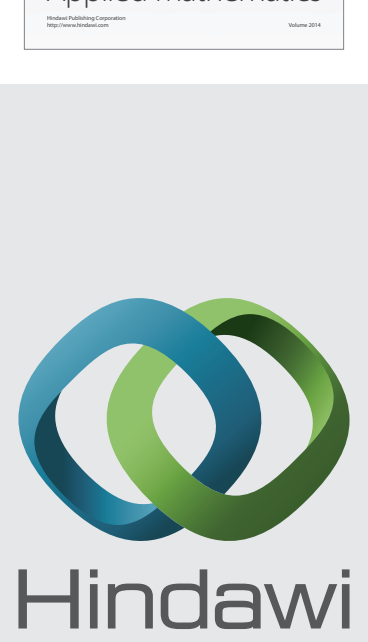

Submit your manuscripts at http://www.hindawi.com
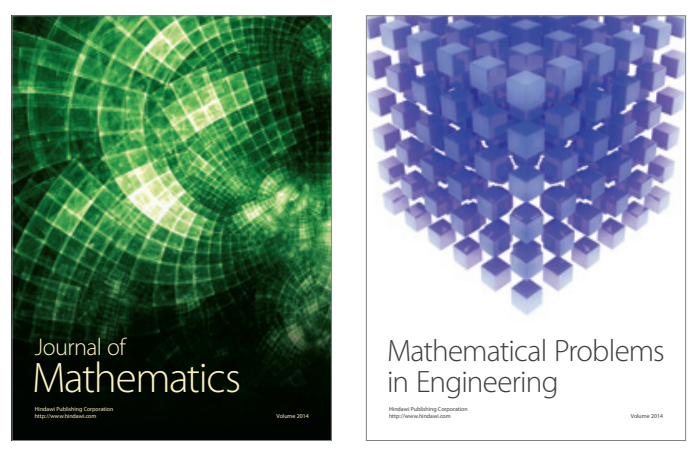

Mathematical Problems in Engineering
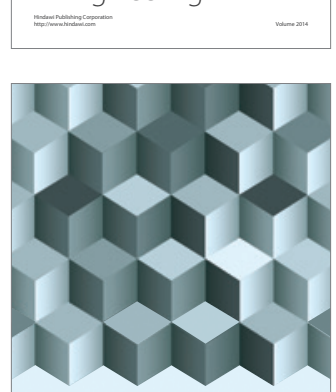

Journal of

Function Spaces
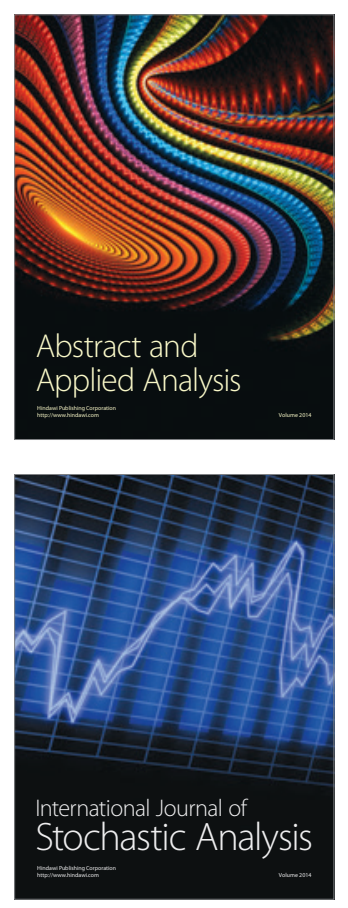

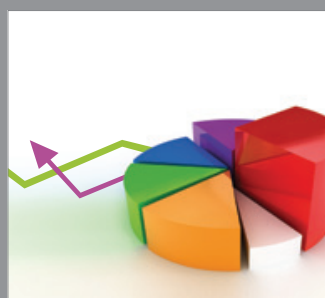

ournal of

Probability and Statistics

Promensencen
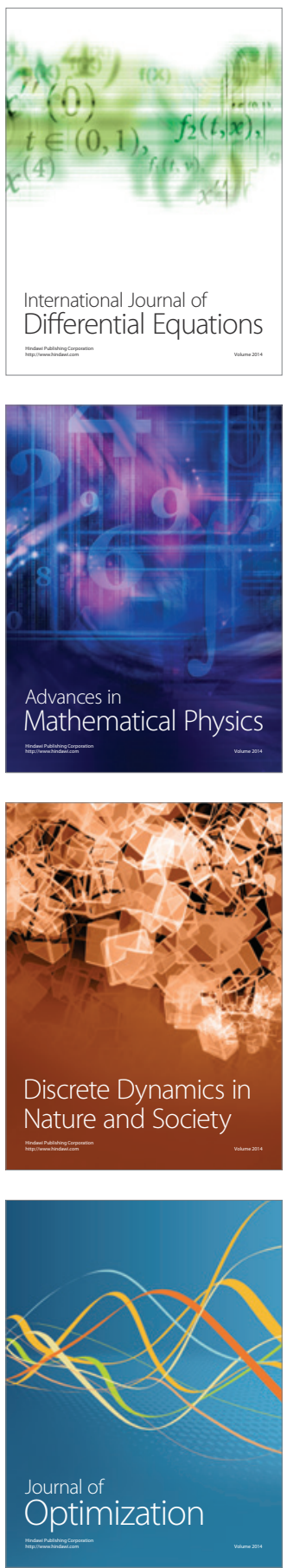